ARTICLE

DOI: $10.1038 / s 41467-018-05887-x$

OPEN

\title{
High-throughput chromatin accessibility profiling at single-cell resolution
}

Anja Mezger ${ }^{1,2}$, Sandy Klemm , Ishminder Mann ${ }^{3}$, Kara Brower ${ }^{4}$, Alain Mir ${ }^{3}$, Magnolia Bostick ${ }^{3}$, Andrew Farmer ${ }^{3}$, Polly Fordyce ${ }^{1,4,5,6}$, Sten Linnarsson (1) ${ }^{2}$ \& William Greenleaf (1) 1,6,7

Here we develop a high-throughput single-cell ATAC-seq (assay for transposition of accessible chromatin) method to measure physical access to DNA in whole cells. Our approach integrates fluorescence imaging and addressable reagent deposition across a massively parallel (5184) nano-well array, yielding a nearly 20-fold improvement in throughput (up to $\sim 1800$ cells/chip, $4-5 \mathrm{~h}$ on-chip processing time) and library preparation cost ( 81申 per cell) compared to prior microfluidic implementations. We apply this method to measure regulatory variation in peripheral blood mononuclear cells (PBMCs) and show robust, de novo clustering of single cells by hematopoietic cell type.

\footnotetext{
${ }^{1}$ Department of Genetics, Stanford University, Stanford, CA 94305, USA. ${ }^{2}$ Department of Medical Biochemistry and Biophysics, Karolinska Institute, Stockholm 17177, Sweden. ${ }^{3}$ Takara Bio USA, Mountain View, CA 94043, USA. ${ }^{4}$ Department of Bioengineering, Stanford University, Stanford, CA 94305, USA. ${ }^{5}$ ChEM-H Institute, Stanford University, Stanford, CA 94305, USA. ${ }^{6}$ Chan Zuckerberg Biohub, San Francisco, CA 94158, USA. ${ }^{7}$ Department of Applied Physics, Stanford University, Stanford, CA 94305, USA. These authors contributed equally: Anja Mezger, Sandy Klemm. Correspondence and requests for materials should be addressed to W.G. (email: wjg@stanford.edu)
} 
central challenge of systems biology is to determine the epigenome of phenotypically distinct cellular states within complex primary tissue. Toward this goal, single-cell chromatin accessibility measurements provide an important epigenetic view of the regulatory landscape within individual cells by capturing the physical accessibility of putative functional elements across the genome ${ }^{1-6}$. Methods for measuring chromatin accessibility at single-cell resolution, however, are low throughput, depth limited, or require complex molecular processing to generate cellular indexing reagents ${ }^{2-5,7}$. For ultra-high throughput accessibility profiling applications, combinatorial indexing approaches ${ }^{2,7}$ offer significant promise, yet these methods capture fewer accessible fragments per cell than single-cell isolation technologies ${ }^{1,3}$ and are not amenable to integration with singlecell microscopy or other multi-omic assays that require whole, live cells. In this report, we describe a high-throughput implementation of single-cell ATAC-seq ${ }^{8}$ (scATAC-seq) that directly integrates fluorescence imaging and provides an extensible foundation for multi-omic epigenetic profiling in single cells.

\section{Results}

Implementation of scATAC-seq on nanoliter-scale wells. We have implemented scATAC-seq in small volumes ( $\mu$ ATAC-seq) using a recently developed nanoliter-scale liquid deposition system (ICELL8 Single Cell System, Takara Bio USA). This approach reduces reagent costs and achieves equal or higher per-cell fragment counts than prior state-of-the-art implementations ${ }^{2,3,7}$. The workflow-illustrated in Figure 1a-is comprising of the following steps: (1) isolated single cells are stained with Hoechst and

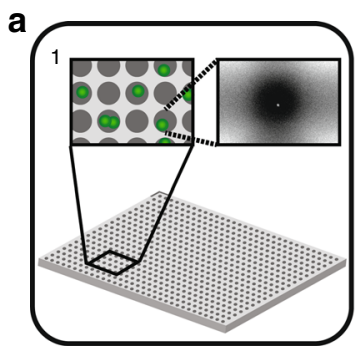

Dispense \& image

b

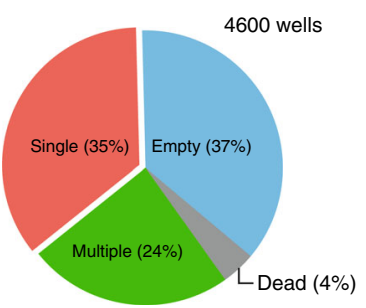

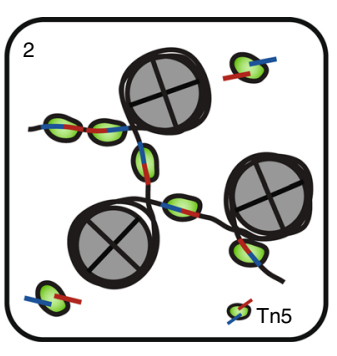

Lyse \& transpose

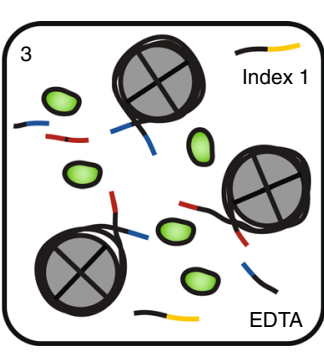

Release transposase \& index 1

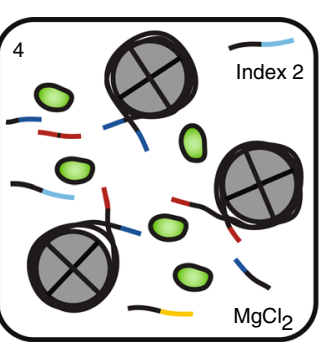

Quench release buffer \& index 2

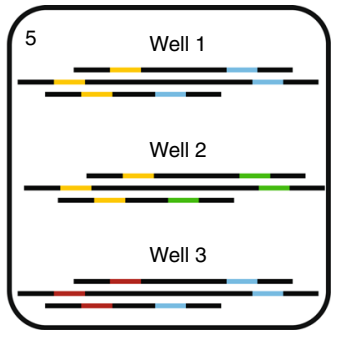

PCR amplification

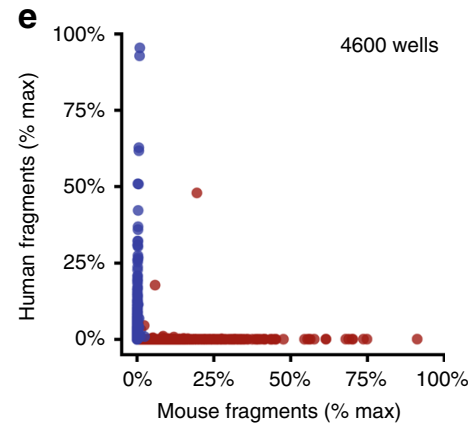

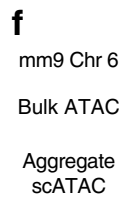
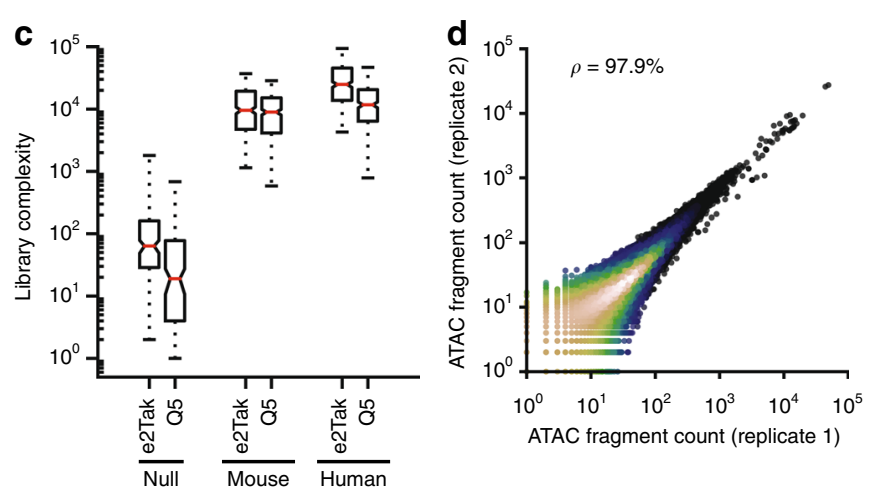

ATAC fragment count (replicate 1)
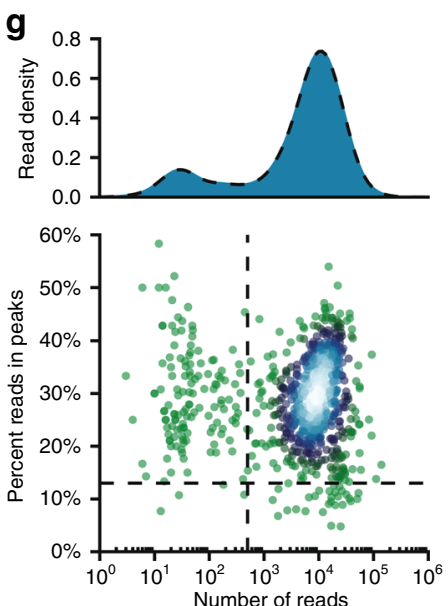

Fig. $1 \mu$ ATAC-seq: a nano-well sCATAC-seq implementation on the ICELL8 platform. a $\mu$ ATAC-seq workflow. $\mathbf{b}$ Distribution of cell counts per well measured by fluorescence microscopy (Hoechst). c $\mu$ ATAC-seq library complexity for null, mouse, and human targeted wells using two separate polymerases (e2Tak and Q5) for well barcoding and amplification ( $n=5000$ wells). For each sample, the box denotes the interquartile range centered at the median (red line), while the whiskers span the 5th and 95th percentile range. d Correlation between nano-well chips processed with either a e2Tak (replicate 1) or Q5 polymerase (replicate 2) across all accessible loci. e Inter-well mixing of mouse and human $\mu$ ATAC-seq fragments. $\mathbf{f}$ Representative population ${ }^{22}$ and single-cell ATAC-seq genome tracks for the Gapdh locus. $\mathbf{g}$ Signal-to-background (percent reads in peaks) as a function of read depth $(n=792)$. Only cells lying in the upper right quadrant (marked by dashed lines) are retained for downstream analysis 
propidium iodide and stochastically loaded under Poisson statistics ( $\sim 1$ cell per well on average) across 5184 wells under active humidity and temperature control; all wells are then imaged via multi-color microscopy to identify those containing a single-live cell; (2) transposition reagents are added to a selected set of wells (e.g., those containing a single live cell) and incubated at $37^{\circ} \mathrm{C}$ for $30 \mathrm{~min}$; (3) the transposition reaction is quenched by incubation with EDTA; (4) $\mathrm{MgCl}_{2}$ is added in equimolar concentration to quench the chelating capacity of EDTA in preparation for subsequent PCR amplification; (5) PCR reagents are added and $\mu$ ATAC-seq fragment libraries are amplified using barcoded primers provided in the prior two steps (see Supplementary Table 1 for reagent loading chart). Following on-chip library construction, indexed $\mu$ ATAC-seq libraries are extracted from all nano-wells by centrifugation, purified, and then further amplified as necessary for sequencing (Methods section).

Benchmarking analysis of $\mu$ ATAC. As an initial test of $\mu$ ATACseq, we loaded samples into 5000 wells across two nano-well ICELL8 chips. On each chip, 200 wells were loaded with PBS (designated null wells); 1150 wells were loaded with mouse embryonic stem cells (mESCs, $\sim 1$ cell per well); and 1150 wells loaded with human lymphoblastoid GM12878 cells $(\sim 1$ cell per well). This yielded a total of 4600 wells targeted with either human or mouse cells across both chips. Imaging of Hoechst and propidium iodide fluorescence revealed the anticipated fraction of wells containing live single cells $(35 \%, 1616$ single cells), consistent with near optimal loading that maximizes the number of single-cell containing wells (Fig. 1b). Barcoded sequencing of each of the 5000 targeted wells revealed $14.3 \times 10^{3}\left(8.1 \times 10^{3}\right)$ median fragments per single human (mouse) cell containing wells $(n=1616)$-reflecting a two orders of magnitude enrichment over null wells (Fig. 1c and Supplementary Figure 1a,b). These library complexities compare favorably with microfluidic cell capture $\left(5.8 \times 10^{3}\right.$ fragments per GM12878 $\left.\mathrm{cell}^{3}\right)$ as well as combinatorial indexing $\left(2.5 \times 10^{3}\right.$ fragments per GM12878 cell $\left.^{7}\right)$ approaches. The $\mu$ ATAC-seq libraries capture both sub-nucleosome as well as nucleosome length fragments, yet, the median fragment length is shorter than that observed using the Fluidigm C1 platform. Consistent with prior bulk and single-cell ATAC-seq libraries, we observe a more than tenfold enrichment for fragments proximal to transcription start sites (TSS) relative to distal regions, reflecting a high fraction of fragments captured within open rather than closed chromatin (Supplementary Figure 2a). Furthermore, we find a high degree of concordance (97.9\%) between nano-well chips even when $\mu$ ATAC-seq fragments are amplified with different polymerases (Fig. 1d). We further tested the deposition fidelity of the ICELL8 platform, observing both human and mouse cells in fewer than $0.2 \%$ of wells (Fig. 1e).

Aggregate single-cell profiles recapitulate population measurements broadly across the accessible genome (Supplementary Figure $2 \mathrm{~b}$ ) as well as specifically at individual genomic loci (Fig. 1f). At single-cell resolution, accessibility profiles are enriched for open chromatin (Fig. 1f, g) in both mESCs $(29 \%$ reads in peaks, Fig. $1 \mathrm{~g})$ and GM12878 cells (22\% reads in peaks, Supplementary Figure 2c). Collectively, these data establish the proposed nano-well implementation as a high-throughput framework for scATAC-seq library construction.

Epigenetic signature distinguishes PBMC types. We next asked whether $\mu$ ATAC-seq epigenetic profiles are sufficient to distinguish cell types within complex primary tissue. For this purpose, we performed $\mu \mathrm{ATAC}$-seq on human peripheral blood mononuclear cells (PBMCs) as well as $\mathrm{B}, \mathrm{T}, \mathrm{CD} 4^{+} \mathrm{T}, \mathrm{CD} 8^{+} \mathrm{T}$, and monocyte cells isolated directly from whole blood (Fig. 2a), yielding 2333 single cells passing all quality control criteria (Methods section). Using ChromVar, a bioinformatic approach described previously ${ }^{9}$, we calculated the relative accessibility of transcription factor (TF) binding motifs in individual cells and found that isolated $\mathrm{B}, \mathrm{T}$, and monocyte cells robustly cluster by cell type (Supplementary Figure 3a). By aggregating fragments within single cells that are proximal to a TF motif, this epigenetic signature captures the variation in putative $\mathrm{TF}$ binding site accessibility across a population of cells ${ }^{9}$. A relatively small fraction of cells are incorrectly assigned to clusters; however, the frequency of these events as well as the random distribution of these cells within apposing clusters both suggest that isolation impurity upstream of the $\mu \mathrm{ATAC}$-seq assay is the primary source of these errors (Supplementary Table 2). PBMC subpopulations co-cluster precisely with the isolated cell types (Fig. 2b, c), showing highly concordant cell type-specific accessibility patterns within appropriate $\mathrm{tSNE}^{10}$ (t-Distributed Stochastic Neighbor Embedding) clusters (Fig. 2c) as well as k-means clustering across highly variable TF binding motif accessibility patterns (Fig. 2b). Consistent with published gene expression data, we find that the PU.1 binding motif is differentially accessible in monocytes and B cells relative to $\mathrm{T}$ cells (Fig. $2 \mathrm{c}$, upper right panel) ${ }^{11,12}$, the $\mathrm{C} /$ EBPa motif is exclusively accessible in monocytes (Fig. 2c, lower left panel) ${ }^{13,14}$, and RUNX1 motif accessibility is appropriately enriched in $\mathrm{T}$ cells-reflecting the broad regulatory role of the RUNX protein family in $\mathrm{T}$ lymphocytes (Fig. 2c, lower right panel) ${ }^{15}$. These results are highly robust to biological (three human blood donors) and technical variation (Supplementary Figure $3 \mathrm{~b}$ ). To further establish the robustness of clustering by cell type, we independently purified $\mathrm{CD}^{+}$and $\mathrm{CD}^{+} \mathrm{T}$ cells and found that these subtypes co-cluster with independently isolated T cells (Fig. 2c, upper left panel). Collectively, these data suggest that $\mu$ ATAC-seq signatures are sufficient for de novo clustering of PBMCs by hematopoietic cell type.

\section{Discussion}

In this report, we have described $\mu \mathrm{ATAC}$-seq-a high-throughput, single-cell chromatin accessibility assay that dramatically reduces per-cell costs, requires only commercially available reagents, provides state-of-the-art data quality, and increases throughput nearly 20 -fold over existing single-cell capture technologies. Single-cell chromatin measurements present a unique experimental challenge since only two DNA templates are present in a diploid cell. Technical sampling noise as well as biological heterogeneity further confound this problem, resulting in a $\sim 10 \%$ observation efficiency of accessible regions in single cells ${ }^{3}$. Consequently, a few hundred cells are typically required to reliably determine the accessibility landscape of each subpopulation within a mixture of cells. Our approach in this work has been to develop an experimental framework for processing more than a thousand of cells in parallel to determine the accessibility of multiple cell types within a complex tissue. In general, nano-well single-cell sequencing approaches such as $\mu$ ATAC-seq are highly extensible, well-suited for multi-omic analysis, and define an important direction for single-cell epigenetic methods development.

\footnotetext{
Methods

Cell culture. All cell lines were grown at $37^{\circ} \mathrm{C}$ with $5 \% \mathrm{CO}_{2}$. GM12878 cells were obtained from the laboratory of Michael Synder (Stanford Univeristy) and were cultured in RPMI 1640 media supplemented with L-glutamine (Thermo Fisher Scientific, MA, USA, Cat. \#11875-085) and 10\% FBS (Thermo Fisher Scientific, Cat. \#10082147); mESC cells (129S1X Castaneous, gift from Howard Chang) were cultured in 15\% FBS (HyClone GE Healthcare Life Sciences, SH30070.03E) supplemented with non-essential amino acids, L-glutamine and Leukemia Inhibitory
} 

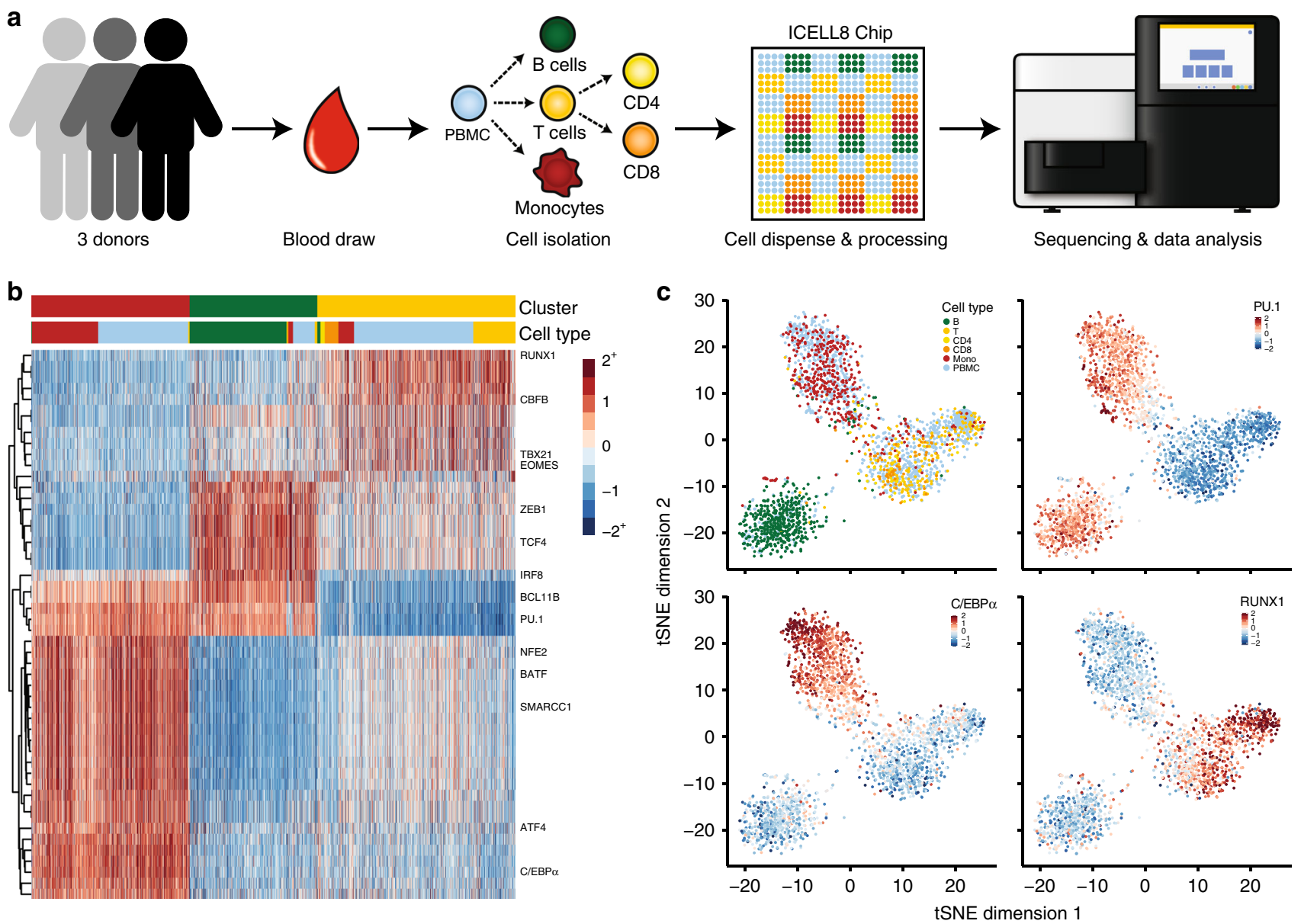

Fig. 2 De novo identification of hematopoietic cell types by $\mu$ ATAC-seq. a Human PBMC isolation and $\mu$ ATAC-seq workflow. $\mathbf{b}$ Hierarchical (TF motifs) and k-means (cells) clustering of accessibility deviation z-scores across 2333 single cells (columns) of the 50 most variable TF motifs (rows). Colors correspond to cell types defined in $\mathbf{a}$. c tSNE visualization of accessibility deviations at TF motifs for cells in $\mathbf{b}$. Cells are either colored by cell type (upper left panel) or by the accessibility deviation $z$-score for the specified TF motif

Factor (LIF, Invitrogen, Cat. \#A35935). Adherent mESCs were washed twice in $1 \mathrm{X}$ PBS and detached using trypsin (Sigma, MO, USA) for 5 min. Cells were diluted in their respective media, collected by centrifugation at $400 \mathrm{~g}$ for $5 \mathrm{~min}$, and then resuspended in media.

Immune cell isolation from whole blood. Monocytes, $\mathrm{T}$ cells, $\mathrm{CD} 4^{+} \mathrm{T}$ cell, $\mathrm{CD} 8^{+}$ $\mathrm{T}$ cells, and $\mathrm{B}$ cells were isolated from whole blood (AllCells, CA, USA) using EasySep Direct Human cell isolation kits (STEMCELL Technologies, MA, USA) according to the manufacturer's protocol. Isolated PBMCs (AllCells, CA, USA) were thawed in RPMI and washed once in media before staining the cells as described below. All human cells were obtained from AllCells with explicit consent to publish data for broad genomic release.

ICELL8 workflow. Cells were stained with Hoechst and propidium iodide using the ReadyProbes Cell Viability Imaging Kit (Thermo Fisher Scientific) for $20 \mathrm{~min}$ in media at $37^{\circ} \mathrm{C}$, then washed twice in cold 0.5X PBS. Cells were counted and dispensed into nano-wells using the SMARTer ${ }^{\mathrm{TM}}$ ICELL8 ${ }^{\circ}$ Single-Cell System (Takara Bio USA, CA, USA, Cat. \#640000) at $25 \mathrm{cells} / \mu \mathrm{l}$ in 0.5X PBS, IX Second Diluent (Takara Bio USA, Cat. \# 640196) and $0.4 \mathrm{U} / \mu \mathrm{l}$ RNase Inhibitor (New England Biolabs [NEB], MA, USA) into a SMARTer ICELL8 250v chip (Takara Bio USA, Cat. \#640183). Control wells containing 1X PBS (25 $\mu$ l) and fiducial mix $(25 \mu l)$ (Takara Bio USA, Cat. \#640196) were included in the source loading plate (see source plate loading chart in Supplementary Table 1). The on-chip deposition volume was $40 \mathrm{nl}$ for all reagent delivery steps. The chips were maintained at $16^{\circ} \mathrm{C}$ or lower between all reagent loading steps. Following cell deposition, chips were sealed with SMARTer ICELL8 imaging film (Takara Bio USA, Cat. \#640014) and centrifuged at $400 \mathrm{~g}$ for $5 \mathrm{~min}$ at $4{ }^{\circ} \mathrm{C}$ and imaged with a $4 \times$ objective using Hoechst and propidium iodide fluorescence. Images were analyzed using automated microscopy image analysis software (CellSelect, Takara Bio USA). Immediately following imaging, the Tn5 transposition mix (2X TD buffer [ $20 \%$ dimethylformamide, $20 \mathrm{mM}$ Tris- $\mathrm{HCl}, \mathrm{pH}$ 7.6, $10 \mathrm{mM} \mathrm{MgCl} 2$ ], $100 \mu \mathrm{l} \mathrm{Tn} 5$ transposase [Nextera DNA Library Prep Kit, Illumina, CA, USA] per ml Tn5 transposition mix, $0.2 \%$ Tween 20, 0.2\% NP40, and 0.02\% Digitonin [Promega, WI, USA]) was dispensed. Chips were then sealed with imaging film, centrifuged at $\sim 3000 \mathrm{~g}$ for $5 \mathrm{~min}$ at $4{ }^{\circ} \mathrm{C}$ and incubated for $30 \mathrm{~min}$ at $37^{\circ} \mathrm{C}$. To index the whole chip, 72 i5 and 72 i7 previously published, custom indices (Supplementary Table 3$)^{3}$ were dispensed at $6.25 \mu \mathrm{M}$ concentration with EDTA and $\mathrm{MgCl}_{2}$, respectively. To release the bound Tn5 transposase, $60 \mathrm{mM}$ EDTA was dispensed together with the i5 indexes. After sealing the chip, it was centrifuged at $3000 \mathrm{~g}$ for $3 \mathrm{~min}$ and incubated for $30 \mathrm{~min}$ at $50^{\circ} \mathrm{C}$. Prior to performing PCR on-chip, the chelating capacity of EDTA was suppressed by dispensing $60 \mathrm{mM} \mathrm{MgCl}$ together with the i7 indices. Chips were then sealed with imaging film, centrifuged, and incubated at room temperature for 5 min. Finally, a PCR mix (5x Q5 [NEB] or e2TAK [Takara Bio USA] reaction buffer, $1 \mathrm{mM}$ dNTPs [Thermo Fisher Scientific], and $100 \mathrm{U} / \mathrm{ml}$ Q5 [NEB] or $50 \mathrm{U} /$ $\mathrm{ml}$ e2TAK polymerase [Takara Bio USA], respectively) was dispensed and 14 cycles of PCR were performed on-chip after sealing with TE Sealing film (Takara Bio

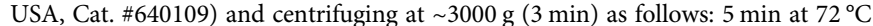
and $30 \mathrm{~s}$ at $98^{\circ} \mathrm{C}$ followed by 14 cycles of $10 \mathrm{sec}$ at $98^{\circ} \mathrm{C}$ and $90 \mathrm{~s}$ (Q5 polymerase) or $150 \mathrm{~s}$ (e2TAK polymerase) at $72^{\circ} \mathrm{C}$, with a final extension of $2 \mathrm{~min}$ at $72^{\circ} \mathrm{C}$. PCR products were extracted by centrifugation at $\sim 3000 \mathrm{~g}$ for $10 \mathrm{~min}$ using the supplied SMARTer ICELL8 Collection Kit (Takara Bio USA). All dispense and sealing steps were followed by centrifugation at $\sim 3000 \mathrm{~g}$ for $3 \mathrm{~min}$. All on-chip thermal cycling was performed using a SMARTer ICELL8 Thermal Cycler (Takara Bio USA).

Off-chip purification and additional amplification. The collected PCR product was purified using MinElute PCR purification columns (Qiagen, Germany) following the manufacturer's instructions. Due to the large sample volume, the PCR product was split across four MinElute columns, eluted in $10 \mu \mathrm{l}$ volumes, and 
subsequently pooled. To remove free PCR primers, which would induce indexswapping during additional rounds of off-chip amplification, we performed two rounds of bead clean-up using Ampure XP beads (Beckman Coulter, CA, USA) in a 1:1.2 ratio. The beads were incubated for $8 \mathrm{~min}$ with the PCR product, washed twice in $70 \%$ ethanol, and eluted in $20 \mu \mathrm{l}$ ultrapure water (Thermo Fisher Scientific). Further amplification was required only for the mouse and human mixing experiment. PBMCs libraries generated on-chip were directly sequenced following column and bead purifications.

The number of required off-chip amplification cycles was determined by running a $20 \mu \mathrm{l}$ qPCR reaction $(2 \mu \mathrm{l} \mathrm{PCR}$ product, $0.5 \mu \mathrm{M}$ oligo C [Illumina P5], $0.5 \mu \mathrm{M}$ oligo D [Ilumina P7], 0.6X SYBR Green I [Thermo Fisher Scientific], and 1X NEBNext High-Fidelity 2X PCR Master Mix [NEB]): $30 \mathrm{~s}$ at $98^{\circ} \mathrm{C}$, followed by 20 cycles of $10 \mathrm{~s}$ at $98^{\circ} \mathrm{C}$ and $30 \mathrm{~s}$ at $63^{\circ} \mathrm{C}$ and $1 \mathrm{~min}$ at $72{ }^{\circ} \mathrm{C}$. The remaining $18 \mu \mathrm{l}$ PCR product was amplified the number of PCR cycles corresponding to $1 / 3$ of the maximum fluorescence intensity. The amplified PCR product was then purified and concentrated using a Qiagen MinElute column.

DNA sequencing. All libraries were sequenced on a NextSeq 500 (Illumina) using the high output v2 kit (Illumina) in $76 \times 8 \times 8 \times 76$ cycle mode, although $38 \mathrm{bp} \times$ $8 \times 8 \times 38$ bp sequencing is sufficient. On average, $\sim 50 \mathrm{~K}$ reads were sequenced per cell. Due to the nature of the sequencing libraries $30-40 \%$ phiX control v3 (Illumina) was spiked in and $1.5 \mathrm{pM}$ were loaded onto the flow cell.

Per cell cost estimate. The per cell library preparation cost is conservatively estimated (assuming only 1200 single cells captured per chip) at 81థ/cell: (1) Takara Bio ICELL8 chip (52屯/cell), (2) Illumina Tn5 (24థ/cell), (3) e2Tak polymerase (4థ/cell), (4) other reagents contribute $<1 \%$ additionally. The additional per cell sequencing cost at the depth used for this report (assuming a 75 cycles NextSeq $500 / 550$ High Output v2 Kit) is approximately $17 \$ /$ cell.

Data analysis. Illumina sequencing reads in BCL format were demultiplexed by single-cell barcode to fastq files using bcl2fastq (Illumina) according to the manufacturer's manual. Reads were trimmed using Cutadapt ${ }^{16}$ (parameters: -a Trans2_rc = CTGTCTCTTATACACATCTCCGAGCCCACGAGACA, Trans1_rc $=$ CTGTCTCTTATACACATCTGACG CTGCCGACGA) and aligned to either the human (hg19) or mouse (mm9) genomes using Bowtie $2^{17}$. Mitochondrial reads were removed prior to downstream analysis. PCR duplicates were identified and removed if either the start or end position was shared with another sequencing read. Library complexity estimates were obtained using the Picard Tools MarkDuplicates utility (https://broad-institute.github.io/picard/), except for emtpy well where too few reads were present for a robust estimate; in the latter case, the library complexity was estimated as the number of unique reads observed. Accessible chromatin regions (peaks) were determined using MACS2 ${ }^{18}$ (parameters: --format BAMPE --nomodel --call-summits --nolambda --keep-dup all) for mouse embryonic stem cells (mESCs) and human lymphoblastoid (GM12878) cells. A previously published accessible peak set for hematopoiesis was used for PBMC, Tand B-cell analysis ${ }^{1}$. Single cells were selected based on imaging using the supplied ICELL8 CellSelect software (Takara Bio USA). Primary PBMCs with fewer than 500 unique (non-mitochondrial) reads or with $<20 \%$ (10-15\% for mESCs and GM12878 cells) of mappable reads lying within peaks were eliminated from subsequent analysis. Bias-corrected deviations in accessibility near transcription factor motifs were calculated using ChromVar ${ }^{9}$. Bias-corrected deviations were linearly transformed to truncated $z$-scores with minimum and maximum values of -2 and 2 , respectively. K-means clustering $(k=3)$ was performed on the 50 most variable transcription factor motifs to assign each single cell to a specific cluster. Transcription factors (rows) were then hierarchically clustered using the ward.D2 agglomeration method ${ }^{19,20}$ within the R pheatmap package ${ }^{21}$, while single cells (columns) were ordered by assigned cluster and cell type (Fig. 2b). Visualizations of clustering and $\mathrm{tSNE}^{10}$ analyses were constructed using R scripts.

\section{Data availability}

The sequencing data that support the findings of this study are available in Figshare under the following DOIs: Metadata: doi: 10.6084/m9.figshare.7006154.v1; Human monocyte cells: doi: 10.6084/m9.figshare.7005707.v1; Human lymphoblast cells (GM12878): doi: 10.6084/m9.figshare.7005713.v1; Human peripheral blood mononuclear cells (PBMCs): doi: 10.6084/m9.figshare.7005752.v1; Mouse embryonic stem cells (mESCs): doi: 10.6084/m9.figshare.7005710.v1; Human CD8 ${ }^{+}$T cells: doi: 10.6084/m9. figshare.7005701.v1; Human CD4+ T cells: doi: 10.6084/m9.figshare.7005698.v1; Human T cells: doi: 10.6084/m9.figshare.7005683.v1; Human B cells: doi: 10.6084/m9. figshare.7005539.v1. All other data are available from the authors upon reasonable request.

Received: 18 April 2018 Accepted: 24 July 2018

Published online: 07 September 2018

\section{References}

1. Corces, M. R. et al. Lineage-specific and single-cell chromatin accessibility charts human hematopoiesis and leukemia evolution. Nat. Genet. 48, 1193-1203 (2016).

2. Cusanovich, D. A. et al. The cis-regulatory dynamics of embryonic development at single-cell resolution. Nature 555, 538-542 (2018).

3. Buenrostro, J. D. et al. Single-cell chromatin accessibility reveals principles of regulatory variation. Nature 523, 486-490 (2015).

4. Pott, S. Simultaneous measurement of chromatin accessibility, DNA methylation, and nucleosome phasing in single cells. eLife 6, e23203 (2017).

5. Jin, W. et al. Genome-wide detection of DNase I hypersensitive sites in single cells and FFPE tissue samples. Nature 528, 142-146 (2015).

6. Thurman, R. E. et al. The accessible chromatin landscape of the human genome. Nature 489, 75-82 (2012).

7. Cusanovich, D. A. et al. Multiplex single cell profiling of chromatin accessibility by combinatorial cellular indexing. Science 348, 910-914 (2015).

8. Buenrostro, J. D., Giresi, P. G., Zaba, L. C., Chang, H. Y. \& Greenleaf, W. J. Transposition of native chromatin for fast and sensitive epigenomic profiling of open chromatin, DNA-binding proteins and nucleosome position. Nat. Methods 10, 1213-1218 (2013).

9. Schep, A. N., Wu, B., Buenrostro, J. D. \& Greenleaf, W. J. chromVAR inferring transcription-factor-associated accessibility from single-cell epigenomic data. Nat. Methods 14, 975-978 (2017).

10. van der Maaten, L., \& Hinton, G. Visualizing data using t-SNE. J. Mach. Learn. Res. 9, 2579-2605 (2008).

11. Chen, H. M. et al. Neutrophils and monocytes express high levels of PU.1 (Spi-1) but not Spi-B. Blood 85, 2918-2928 (1995).

12. Lloberas, J., Soler, C. \& Celada, A. The key role of PU.1/SPI-1 in B cells, myeloid cells and macrophages. Immunol. Today 20, 184-189 (1999).

13. Di Tullio, A. et al. CCAAT/enhancer binding protein alpha (C/EBP(alpha))induced transdifferentiation of pre-B cells into macrophages involves no overt retrodifferentiation. Proc. Natl Acad. Sci. USA 108, 17016-17021 (2011).

14. Laiosa, C. V., Stadtfeld, M., Xie, H., de Andres-Aguayo, L. \& Graf, T. Reprogramming of committed $\mathrm{T}$ cell progenitors to macrophages and dendritic cells by C/EBP alpha and PU.1 transcription factors. Immunity 25, 731-744 (2006)

15. Kohu, K. et al. Pleiotropic roles of runx transcription factors in the differentiation and function of T lymphocytes. Curr. Immunol. Rev. 4, 101-115 (2008).

16. Martin, M. Cutadapt removes adapter sequences from high-throughput sequencing reads. EMBnet J. 17, 10 (2011).

17. Langmead, B. \& Salzberg, S. L. Fast gapped-read alignment with Bowtie 2. Nat. Methods 9, 357-359 (2012).

18. Zhang, Y. et al. Model-based analysis of ChIP-Seq (MACS). Genome Biol. 9 , R137 (2008).

19. Ward, J. H. Hierarchical grouping to optimize an objective function. J. Am. Stat. Assoc. 58, 236-244 (1963).

20. Murtagh, F. \& Legendre, P. Ward's hierarchical agglomerative clustering method: which algorithms implement ward's criterion? J. Classif. 31, 274-295 (2014).

21. Kolde, R. Pheatmap: Pretty Heatmaps. (R package, version 1.0.8, 2015). Available at: https://CRAN.R-project.org/package=pheatmap.

22. $\mathrm{Xu}$, J. et al. Landscape of monoallelic DNA accessibility in mouse embryonic stem cells and neural progenitor cells. Nat. Genet. 49, 377-386 (2017).

\section{Acknowledgements}

A. Mezger is supported by the Swedish Research Council (grant 2015-06403). S.K. is supported by a T32 Ruth L. Kirschstein National Research Service Award (Institutional Training Grant in Genome Science NIH 5 T32 HG000044). This work was supported by NIH (P50HG007735 and UM1HG009442 and U19AI057266 to W.G.), the Rita Allen Foundation (to W.G.), the Baxter Foundation Faculty Scholar Grant, and the Human Frontiers Science Program grant RGY006S (to W.G.), and the Joint Institute for Metrology in Biology. W.G. is a Chan Zuckerberg Biohub investigator and acknowledges grants 2017-174468 and 2018-182817 from the Chan Zuckerberg Initiative.

\section{Author contributions}

A. Mezger, S.K., S.L., and W.G. conceived the study and designed all experiments; A. Mezger and S.K. analyzed the data and performed all experiments with the help of I.M.; K.B. assisted with imaging analysis; A. Mir, M.B., A.F., P.F., and W.G. supervised the study; A. Mezger, S.K., and W.G wrote the manuscript with input from all authors.

\section{Additional information}

Supplementary Information accompanies this paper at https://doi.org/10.1038/s41467 018-05887-x. 
Competing interests: I.M., A. Mir, M.B., and A.F. are employees at Takara Bio USA, Inc. S.K. presented the described work on behalf of Takara Bio USA, Inc. at the Advances in Genome Biology and Technology General Meeting (2018), but has no financial interest in this work. W.G. is a scientific co-founder of Epinomics and a consultant for 10X genomics. Stanford University has filed a provisional patent application (US20160060691A1; Status: Components are pending) on the ATAC-seq methods described and W.G. is named as an inventor. A. Mezger, K.B., P.F., and S.L. declare no competing interests.

Reprints and permission information is available online at http://npg.nature.com/ reprintsandpermissions/

Publisher's note: Springer Nature remains neutral with regard to jurisdictional claims in published maps and institutional affiliations. (c) (i) Open Access This article is licensed under a Creative Commons Attribution 4.0 International License, which permits use, sharing, adaptation, distribution and reproduction in any medium or format, as long as you give appropriate credit to the original author(s) and the source, provide a link to the Creative Commons license, and indicate if changes were made. The images or other third party material in this article are included in the article's Creative Commons license, unless indicated otherwise in a credit line to the material. If material is not included in the article's Creative Commons license and your intended use is not permitted by statutory regulation or exceeds the permitted use, you will need to obtain permission directly from the copyright holder. To view a copy of this license, visit http://creativecommons.org/ licenses/by/4.0/.

(c) The Author(s) 2018 